CASE REPORT

\title{
Malignant prolactinoma: case report and review of the literature
}

\author{
Marleen Kars, Ferdinand Roelfsema, Johannes A Romijn and Alberto M Pereira \\ Department of Endocrinology, Leiden University Medical Center, Albinusdreef 2, PO Box 9600, 2300 RC Leiden, The Netherlands \\ (Correspondence should be addressed to M Kars; Email: m.kars@lumc.nl)
}

\begin{abstract}
Pituitary carcinomas are extremely rare. In general, the initial clinical, biochemical, and histological characteristics are of minimal utility in distinguishing benign adenomas from pituitary carcinomas. We describe a 63-year-old woman with a macroprolactinoma, who presented with diplopia and blurred vision. This unusual initial presentation and the subsequent aggressive clinical course, with diffuse local and distant intramedulary metastases, prompted us in retrospect to make a detailed analysis of the therapeutic interventions and histology. In addition, we reviewed all available literature on published cases of malignant prolactinoma and detailed their epidemiological, clinical, and histopathological characteristics. In brief, it is postulated that pituitary carcinomas arise from the transformation of initially large, but benign, adenomas. Unusual and/or atypical clinical manifestations appear to occur more frequently. In vivo, the development of dopamine agonist resistance in invasive macroprolactinoma is indicative of malignancy and should prompt the clinician to perform a biopsy of the tumor. For pituitary tumors that exhibit high mitotic activity, increased Ki67 and/or p53 immunoreactivity, it may be useful to denote these tumors as 'atypical' prolactinomas to raise the possibility of future malignant development.
\end{abstract}

European Journal of Endocrinology 155 523-534

\section{Introduction}

Although pituitary tumors are relatively common, occurring in approximately $10-20 \%$ of normal subjects on autopsy or magnetic resonance imaging (MRI), the incidence of pituitary carcinoma is extremely low (1). To date, a total of approximately 140 cases have been reported, one third of them being malignant prolactinomas (2). The histological, clinical, and biochemical characteristics are reported to be of minimal utility in distinguishing benign from malignant tumors, unless (distant) metastases have developed. Presently, it is postulated that pituitary carcinomas arise from the transformation of initially large but benign adenomas (1). The arguments are based on observations that the initial presentation is not different from other macroadenomas, the long-duration needed for the transformation into carcinoma, and the increasing accumulation of genetic aberrations (3). We describe a patient with a malignant prolactinoma, whose unusual initial presentation and clinical course prompted us, in retrospect, to make a detailed analysis of the case with respect to the therapeutic interventions and histology. For comparison, we reviewed all published cases of malignant prolactinoma and detailed their epidemiological, clinical, biochemical, and histological characteristics.

\section{Case report}

A 63-year-old woman, who presented with diplopia and blurred vision, was diagnosed with a macroprolactinoma in 1998. On neurological examination, ptosis of the right eye was present, together with abducens palsy and impaired convergence. Furthermore, bitemporal hemianopsia was present (Fig. 1). Prolactin concentration was increased 20-fold: $490 \mu \mathrm{g} / \mathrm{l}$ (normal value $<22 \mu \mathrm{g} / \mathrm{l})$. MRI showed a pituitary mass with a diameter of $2.5 \mathrm{~cm}$, extending into the right sphenoidal sinus as well as the cavernous sinus and compressing the temporal lobe (Fig. 2). Therapy was initiated with bromocriptine ( $1.25 \mathrm{mg}$ t.i.d) resulting in normalization of the visual fields and decrease in prolactin levels to $56 \mu \mathrm{g} / \mathrm{l}$ within a few months.

The visual field defects recurred and prolactin levels increased to $206 \mu \mathrm{g} / \mathrm{l}$ (Fig. 3), 6 months later. Therefore, bromocriptine treatment was switched to quinagolide (up to $300 \mu \mathrm{g} /$ day). Nonetheless, in January 2000, MRI showed progression of the pituitary tumor with encasement of the internal carotid artery and compression of the optic chiasm. The macroprolactinoma did not react satisfactory to medical treatment, even with cabergoline, which was stopped, since prolactin levels progressively increased in the presence of further progression of tumor 


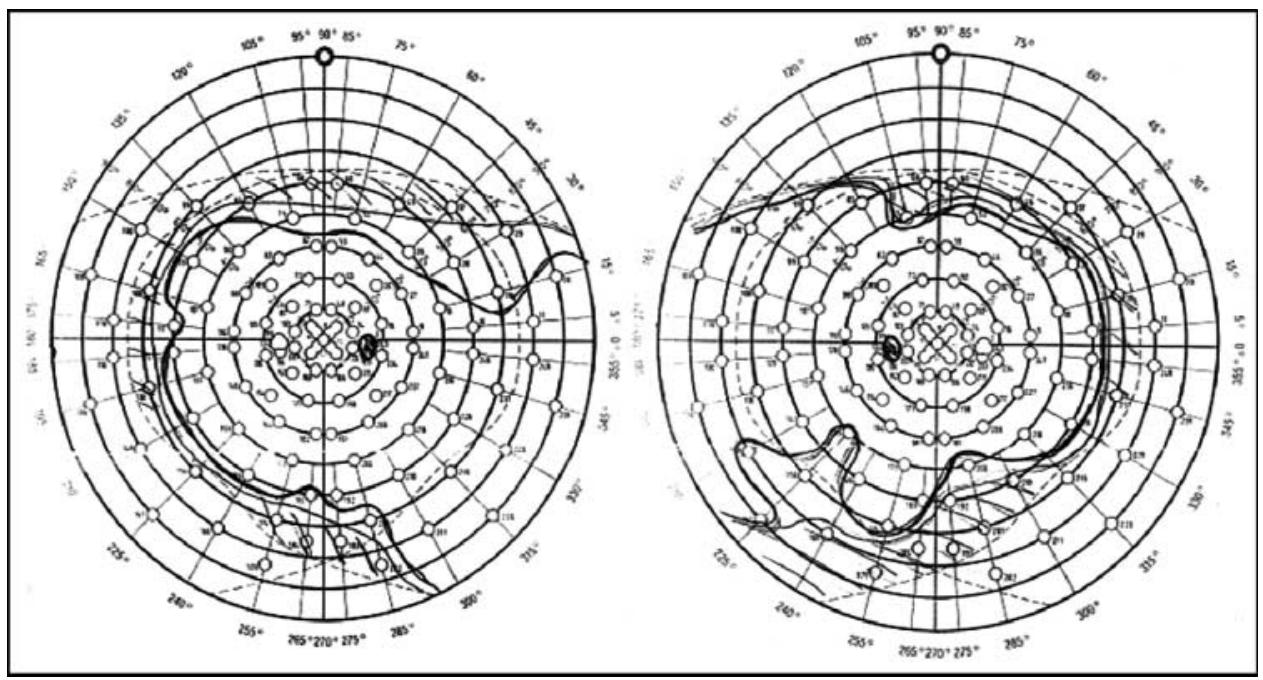

Figure 1 Visual field investigation in October 1999, revealing bitemporal hemianopsia.

growth. Furthermore, she developed progressive anterior pituitary insufficiency (de novo adrenocorticotropic hormone and thyroid-stimulating hormone deficiency) and the visual field defects increased. Therefore, she was operated in April 2000. Decompression of the optic chiasm was performed via transcranial route. Histological investigation of the tumor revealed positive immunostaining for prolactin without mitotic activity, but high Ki-67 (MIB-1) labeling index (10-15\%). Fractionated conventional radiotherapy was administered by a linear accelerator $(6 \mathrm{MeV})$ in a total dose of 54 Gray (Gy) in June 2000. Thereafter, prolactin concentrations decreased gradually without dopaminergic therapy from $445 \mu \mathrm{g} / \mathrm{l}$ in June 2000 to a nadir of $33 \mu \mathrm{g} / \mathrm{l}$ in February 2001 (Fig. 3). The effect on tumor volume in response of radiotherapy was evaluated 8 months after radiotherapy with MRI. A slight reduction in tumor volume was noted. Encasement of the internal carotid artery persisted.

Serum prolactin levels started to rise again in August 2001. MRI of the brain did not reveal progression of the tumor, but the rise in prolactin concentration proved to be due to metastases in the spinal cord (Fig. 4), which was confirmed with epidepride (dopamine D2 receptor) scintigraphy (Fig. 5). Laminectomy was performed in December 2001 because of compression of the myelum in the sacral region, followed by fractionated radiotherapy $(6 \times 4 \mathrm{~Gy})$ from L5 to S5 in February 2002. Histological examination confirmed a prolactin-producing metastasis.

Subsequently, the patient developed extensive metastases throughout the spinal cord. Therefore, the spinal cord was irradiated with fractionated radiotherapy aimed at C2 to L4 with a total dose of $40 \mathrm{~Gy}$ in June 2002 to relieve pain and prevent paralysis from compression of the myelum.

In August 2002, she developed progressive ptosis of the right eye and facial paralysis due to infiltration of the tumor into the orbital cavity. Repeat radiotherapy to the skull (total dose of $25 \mathrm{~Gy}$ ) was given in September 2002, resulting only in partial improvement of visual disturbances. However, prolactin concentrations continued to increase (Fig. 3) to a final concentration of $6000 \mu \mathrm{g} / \mathrm{l}$ in May 2003, 1 month before she died at home. Autopsy was not performed.

\section{Discussion}

Pituitary carcinomas are considered to arise from the transformation of initially large, but benign, adenomas (1). This notion is based on observations that

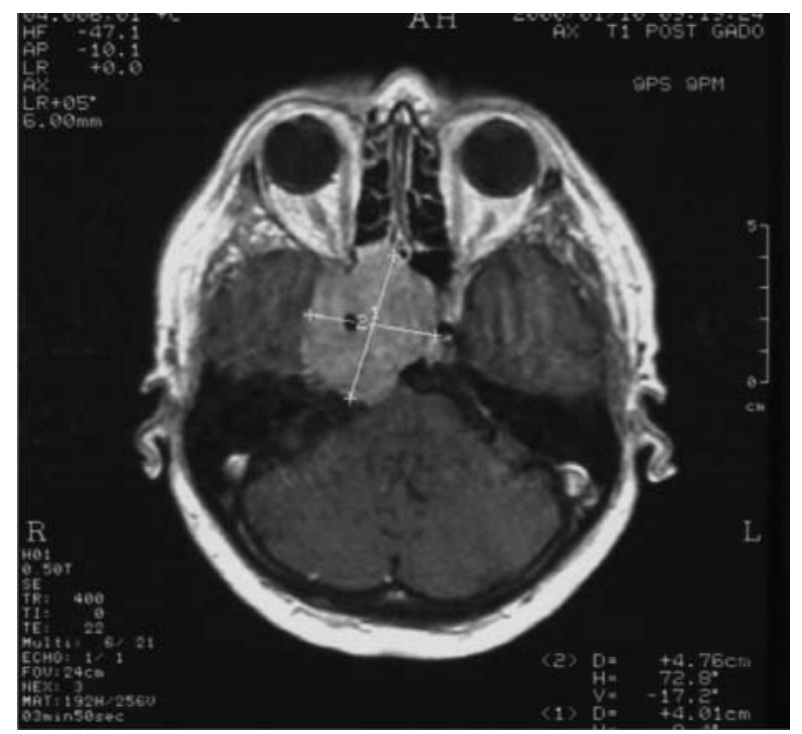

Figure 2 Magnetic resonance image (axial T1-weighted image) obtained in April 1999, demonstrating a pituitary mass of $2.5 \mathrm{~cm}$, invading the right sphenoidal and cavernous sinus (Hardy classification IV-E (47)), and encasement of internal carotid artery. 


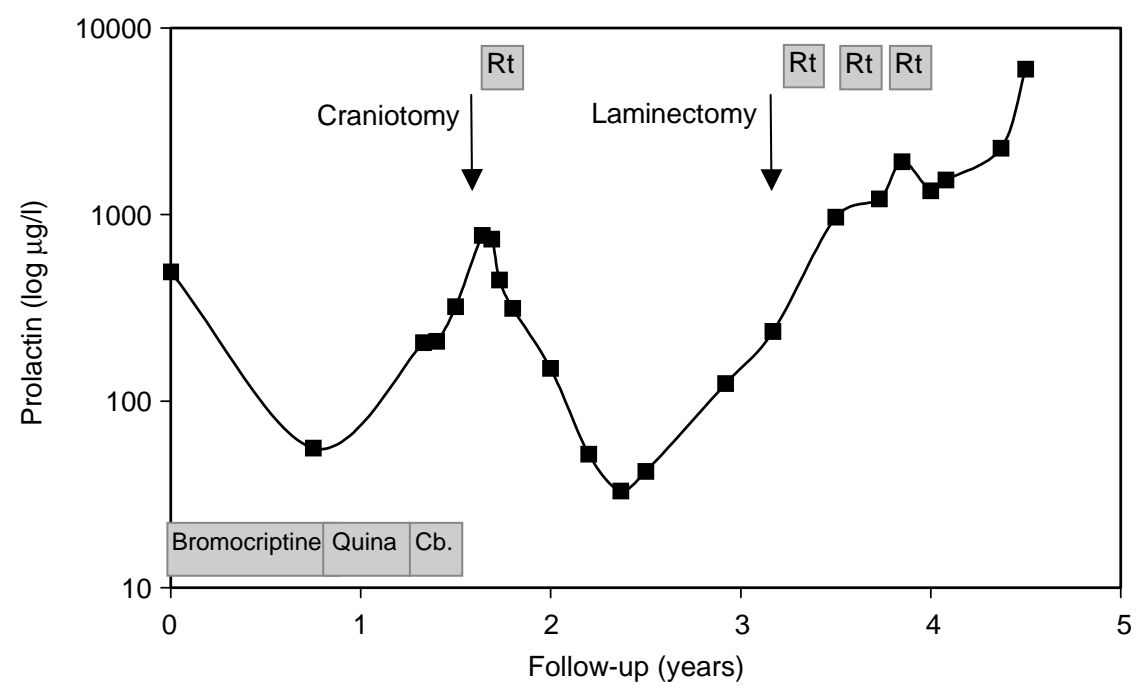

Quina.= quinagolide, $\mathrm{Cb}=$ cabergoline, $\mathrm{Rt}=$ radiotherapy
Figure 3 Serum prolactin concentrations from October 1998 to April 2003 (normal value $<22 \mu \mathrm{g} / \mathrm{l}$ ). demonstrate that the initial presentation of pituitary carcinomas does not differ from other (invasive) macroadenomas, the long-duration needed for transformation into a carcinoma, and the progressive accumulation of genetic aberrations (3). The present case of malignant prolactinoma is consistent with many, but not all, of these observations. This gave us an opportunity to compare carefully the data of our patient and review the intriguing features associated with malignant transformation of pituitary prolactinomas.

To date, only 47 cases of malignant prolactinoma have been reported, summarized in Table 1 (4-41). The first report of a patient with malignant prolactinoma was in 1981 by Martin et al. (4). Of the reported cases, $65 \%$ were male and the mean age at presentation was 44 years with a range of $14-70$ years (Table 2). The presenting symptoms were related to hyperprolactinemia in $35 \%$ of the reported cases, including amenorrhea, galactorrhea, impotency, and decreased libido. At presentation, $71 \%$ of the patients had symptoms of local compression, such as headache and bitemporal hemianopsia. Only five other patients presented with ptosis $(27,40)$, diplopia (34), oculomotor paresis (17), or lateral rectus paresis (13). Diabetes insipidus was a feature in only one patient (12). The treatment modalities after diagnosis were transsphenoidal or transcranial surgery $(96 \%)$, radiotherapy $(79 \%)$, chemotherapy (2\%), and dopamine agonists (DA) in $65 \%$ of the cases (Table 3). A study by Isobe et al. shows that, in particular, large prolactinomas are very difficult to control with radiation doses between 50 and $60 \mathrm{~Gy}$ (42). Therefore, even benign prolactinomas do not respond very well to radiotherapy. The effect of radiotherapy on malignant prolactinoma has not been systematically documented. Therefore, a small response to radiotherapy, as in our case, cannot be interpreted as an indication of the malignant nature of the tumor.
The presentation of our patient with diplopia and blurred vision is a very unusual manifestation of pituitary macroadenoma. Such a presentation is most frequently associated with pituitary apoplexia. In the

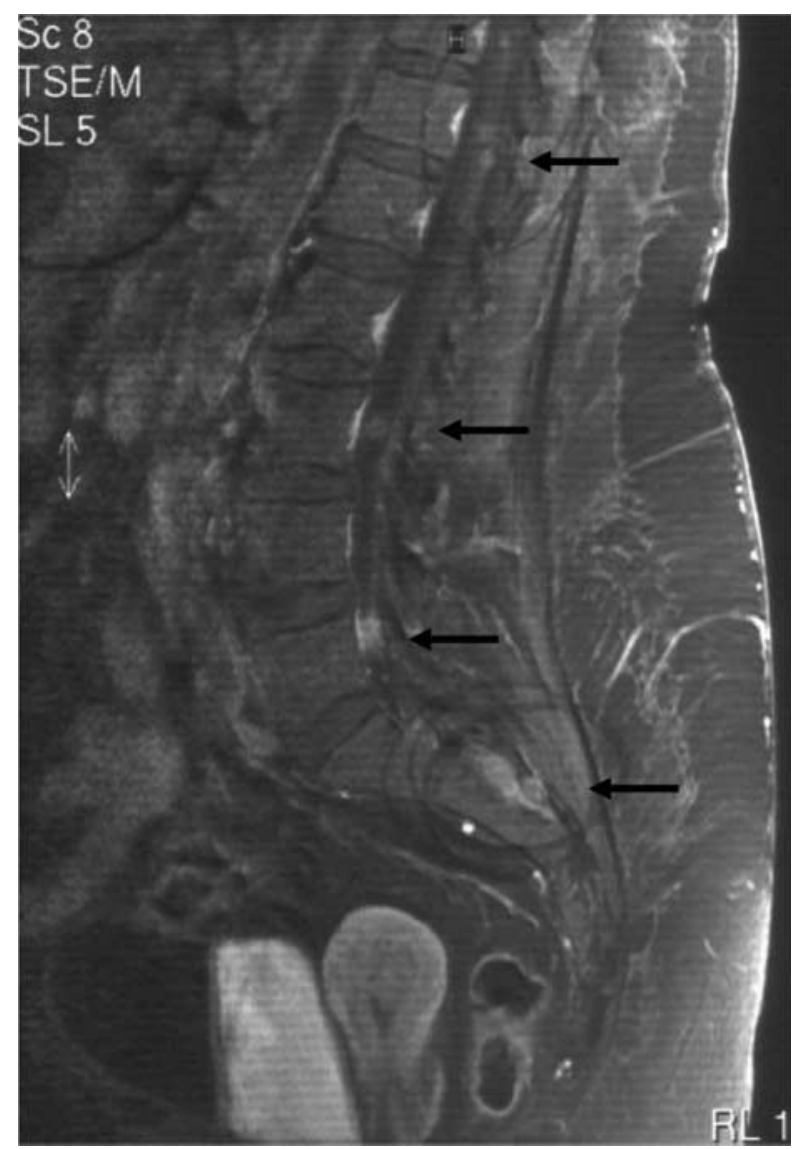

Figure $4 \mathrm{MRI}$ (sagittal T1-weighted image) obtained in November 2001, demonstrating spinal lesions (arrows) in the lumbar and sacral region. 


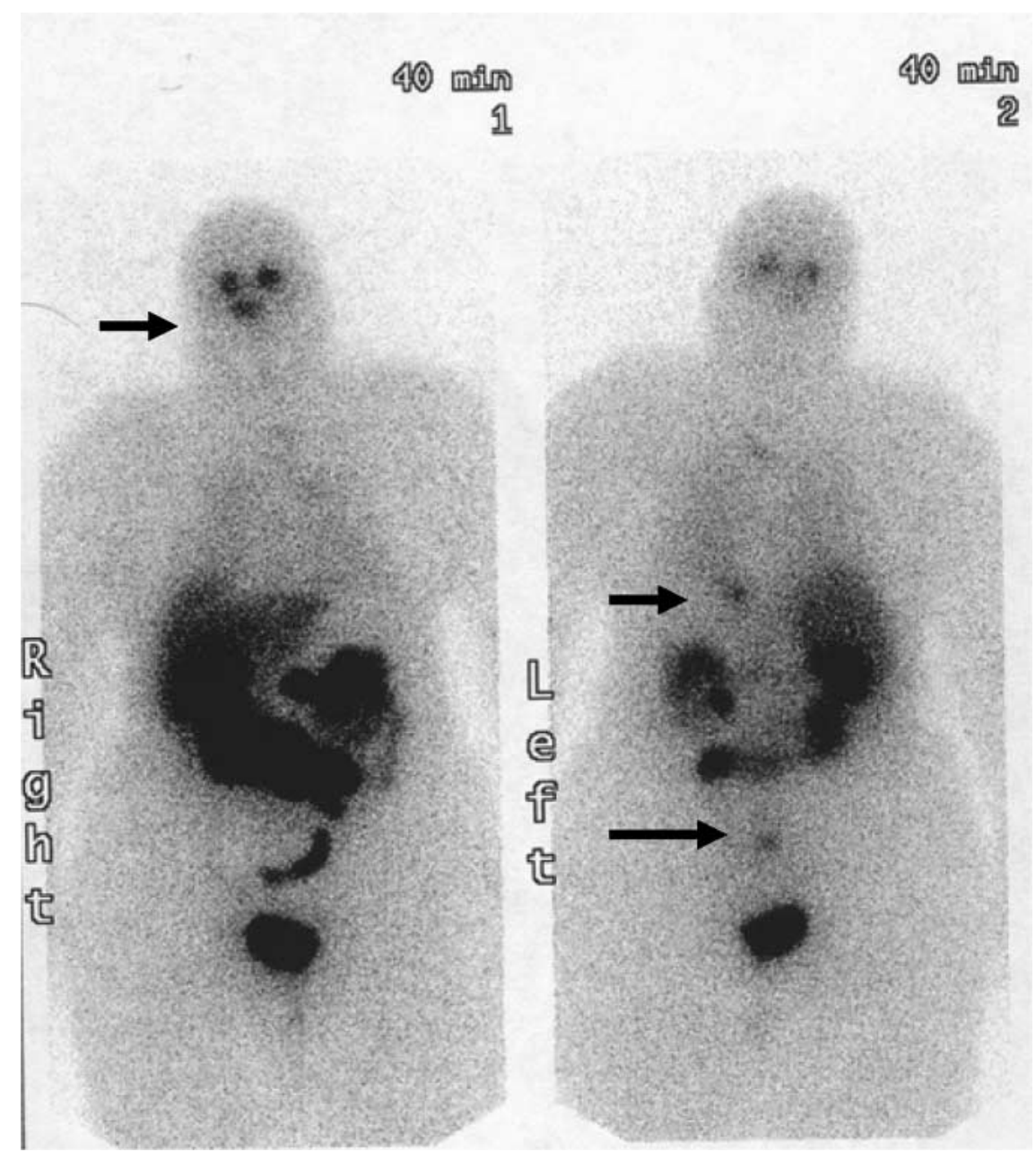

Figure 5 Total-body scintigraphy after $\left[{ }^{123} \mathrm{I}\right]$ epidepride injection in December 2001. Physiological accumulation of activity in basal ganglia, liver, kidneys, bowel, and urinary bladder. Anterior view (left image): intracranial accumulation of the isotope reflecting the macroprolactinoma (arrow). Posterior view (right image): multiple accumulations of the isotope reflecting multiple metastases in the spine (arrows).

absence of apoplexia, nerve paralysis is strongly suggestive of compression or infiltration of the nerve, secondary to the high proliferative activity of the tumor. The presentation with diplopia as a result of oculomotor nerve paralysis has been reported previously in only one other case(17). In the present case, oculomotor nerve paralysis was due to tumoral orbital invasion (Fig. 2). Orbital invasion of a pituitary adenoma is very uncommon, being reported in only 16 patients, only 2 of whom manifested diplopia (43). Thus, in retrospect, the initial clinical and radiological presentation was highly indicative for an adenoma with high infiltrative potency.

Kaltsas et al. described histological and immunohistochemical parameters that predict the biological behavior of pituitary tumors $(1,3)$. Histological parameters associated with an atypical or aggressive behavior of the adenoma are cellular atypia, nuclear pleomorphism, more than two mitotic figures per ten high-powered fields, a proliferative index of Ki-67 more than 3\%, positive p53 immunoreactivity, and invasion.
They are also called atypical parameters. Histological investigation of the tissue initially obtained by surgery, biopsy, or autopsy of the prolactinoma revealed a benign classification in $37 \%$ and an atypical classification in $40 \%$ (Table 3). In the remaining $23 \%$ of cases, no documentation of the histological findings was given. The histological investigation in our case demonstrated a prolactinoma with a high proliferative index, such as nuclear pleomorphism and high Ki-67 labeling index. Estimation of the cell cycle-specific antigen Ki-67, using the MIB-1 antibody, has been demonstrated to correlate best with invasiveness and probably prognosis (1). Malignant and invasive tumors exhibit much higher Ki67 labeling indices than benign adenomas (11.9 vs 4.66 vs $1.37 \%$ respectively) (44), although there is considerable case-to-case variability (1). Others even suggested that an increased Ki-67 labeling index is associated with secondary resistance or escape to DA treatment (45).

The time interval between the onset of symptoms at presentation and subsequent metastases in the 
Table 1 List of previously published cases of malignant prolactinoma.

\begin{tabular}{|c|c|c|c|c|c|c|c|c|c|}
\hline $\begin{array}{l}\text { Author, year of } \\
\text { publication }\end{array}$ & $\begin{array}{l}\text { Age/ } \\
\text { sex }\end{array}$ & $\begin{array}{l}\text { Primary } \\
\text { treatment }^{\mathrm{a}}\end{array}$ & $\begin{array}{l}\text { PA primary } \\
\text { tumor }\end{array}$ & $\begin{array}{l}\text { Time interval } \\
\text { diagnosis - } \\
\text { metastases } \\
\text { (years) }\end{array}$ & Metastatic sites & $\begin{array}{l}\text { Treatment of } \\
\text { metastases }^{a}\end{array}$ & PA metastases & $\begin{array}{c}\text { Cause of } \\
\text { death }\end{array}$ & $\begin{array}{c}\text { Time interval } \\
\text { diagnosis - } \\
\text { death (years) }\end{array}$ \\
\hline Martin, 1981 & $31 / F$ & TSS, Rt, CT, DA, Rt & $\begin{array}{l}\text { Pleomorphism, } \\
\text { rare mitotic } \\
\text { figures }\end{array}$ & 5.5 & Cerebellum & CT & $\begin{array}{l}\text { Numerous mitotic } \\
\text { figures }\end{array}$ & $\begin{array}{l}\text { Death due to } \\
\text { disease pro- } \\
\text { gression }\end{array}$ & 8.5 \\
\hline Cohen, 1983 & 70/M & - & No mitotic figures ${ }^{b}$ & 3.5 & $\begin{array}{l}\text { Cerebellopontine } \\
\text { angle }\end{array}$ & - & No mitotic figures ${ }^{b}$ & $\begin{array}{l}\text { Pulmonary } \\
\text { edema, cir- } \\
\text { culatory } \\
\text { shock }\end{array}$ & 3.58 \\
\hline U, 1984 & $63 / M$ & $\mathrm{CT}, \mathrm{Rt}$ & Mitosis $3 / 20 \mathrm{HPF}$ & 6 & Cerebrum & $\mathrm{CT}, \mathrm{Rt}, \mathrm{DA}$ & $\begin{array}{l}\text { Mitosis } 11 / 20 \\
\text { HPF, pleo- } \\
\text { morphic }\end{array}$ & $\begin{array}{l}\text { Pulmonary } \\
\text { embolus }\end{array}$ & 6.25 \\
\hline Gasser, 1985 & $28 / M$ & $\mathrm{CT}, \mathrm{Rt}, \mathrm{DA}$ & $\begin{array}{l}\text { Mitosis, pleo- } \\
\text { morphism }\end{array}$ & 9 & Cerebrum & $\mathrm{CT}, \mathrm{DA}, \mathrm{Rt}, \mathrm{CT}$ & $\begin{array}{l}\text { Mitosis, pleo- } \\
\text { morphism; } \\
\text { tumor cells in } \\
\text { subarachnoidal } \\
\text { space and in } \\
\text { venous blood } \\
\text { channels }\end{array}$ & $\begin{array}{l}\text { Death due to } \\
\text { disease pro- } \\
\text { gression }\end{array}$ & 12 \\
\hline Landgraf, 1985 & $44 / F$ & $\mathrm{CT}, \mathrm{Rt}, \mathrm{DA}$ & Benign & 4 & $\begin{array}{l}\text { Cerebellum, spinal } \\
\text { cord }\end{array}$ & $\begin{array}{l}\text { LT, Rt, DA, } \\
\text { chemo, octa- } \\
\text { peptide- } \\
\text { somato- } \\
\text { statin, } \\
\text { chemo }\end{array}$ & Benign & $\begin{array}{l}\text { Death due to } \\
\text { disease pro- } \\
\text { gression }\end{array}$ & 5.5 \\
\hline Plangger, 1985 & $28 / M$ & $\mathrm{CT}, \mathrm{Rt}$ & Benign & 9 & $\begin{array}{l}\text { Cerebrum, subar- } \\
\text { achnoid nodules }\end{array}$ & CT, Rt, DA, CT & Mitotic figure rare & $\begin{array}{l}\text { Still alive at } \\
\text { publication }\end{array}$ & \\
\hline Scheithauer, 1985 & $52 / F$ & Rt, CT, TSS(2x), DA & Mitotic figures rare & 11 & $\begin{array}{l}\text { Cerebrum, } \\
\text { vertebrae, occi- } \\
\text { pital bone, ribs }\end{array}$ & $\mathrm{DA}, \mathrm{Rt}(2 \mathrm{x})$ & Mitotic figure rare ${ }^{c}$ & $\begin{array}{l}\text { Death due to } \\
\text { disease pro- } \\
\text { gression }\end{array}$ & 13.5 \\
\hline Von Werder, 1985 & $43 / F$ & CT, Rt, DA & Not documented & 4 & Spinal cord & $\mathrm{DA}, \mathrm{LT}, \mathrm{Rt}, \mathrm{DA}$ & Not documented & Unknown & \\
\hline Muhr, 1988 & $14 / \mathrm{M}$ & $\mathrm{CT}, \mathrm{Rt}$ & Benign & 12 & $\begin{array}{l}\text { Cerebellum, fron- } \\
\text { tal lobe }\end{array}$ & Surgery, DA & Mitosis & $\begin{array}{l}\text { Still alive at } \\
\text { publication }\end{array}$ & \\
\hline Atienza, 1991 & $34 / \mathrm{M}$ & $\mathrm{DA}, \mathrm{CT}(2 \mathrm{x}), \mathrm{Rt}, \mathrm{DA}$ & No mitotic figures & 4 & $\begin{array}{l}\text { Spinal cord, } \\
\text { pulmonary } \\
\text { nodules }\end{array}$ & DA, Rt, TSS & $\begin{array}{l}\text { Mitosis } 2 / 10 \mathrm{HPF} \text {, } \\
\text { vascular inva- } \\
\text { sion }\end{array}$ & $\begin{array}{l}\text { Death due to } \\
\text { disease pro- } \\
\text { gression }\end{array}$ & 5.5 \\
\hline \multirow[t]{2}{*}{ Popovic, 1991} & $47 / M$ & $\mathrm{DA}, \mathrm{CT}(2 \mathrm{x}), \mathrm{Rt}$ & Mitosis 6/HPF & 2 & $\begin{array}{l}\text { Dura, cerebrum, } \\
\text { cerebellum }\end{array}$ & CT & Mitosis 6/HPF & $\begin{array}{l}\text { Gastrointes- } \\
\text { tinal bleeding }\end{array}$ & 2.02 \\
\hline & $56 / F$ & TSS, Rt & Mitosis $5 / 10 \mathrm{HPF}$ & 12 & $\begin{array}{l}\text { Roof fourth ventri- } \\
\text { cle, cerebrum, } \\
\text { spinal cord }\end{array}$ & CT & Mitosis $5 / 10 \mathrm{HPF}$ & $\begin{array}{l}\text { Acute pulmon- } \\
\text { ary edema, } \\
\text { Staphylococ- } \\
\text { cus aureus } \\
\text { septicemia }\end{array}$ & 12.33 \\
\hline
\end{tabular}




\begin{tabular}{|c|c|c|c|c|c|c|c|c|c|}
\hline $\begin{array}{l}\text { Author, year of } \\
\text { publication }\end{array}$ & $\begin{array}{l}\text { Age/ } \\
\text { sex }\end{array}$ & $\begin{array}{l}\text { Primary } \\
\text { treatment }^{\mathrm{a}}\end{array}$ & $\begin{array}{l}\text { PA primary } \\
\text { tumor }\end{array}$ & $\begin{array}{l}\text { Time interval } \\
\text { diagnosis - } \\
\text { metastases } \\
\text { (years) }\end{array}$ & Metastatic sites & $\begin{array}{l}\text { Treatment of } \\
\text { metastases }^{a}\end{array}$ & PA metastases & $\begin{array}{l}\text { Cause of } \\
\text { death }\end{array}$ & $\begin{array}{l}\text { Time interval } \\
\text { diagnosis - } \\
\text { death (years) }\end{array}$ \\
\hline Berezin, 1992 & $32 / F$ & $\mathrm{CT}, \mathrm{Rt}$ & Benign & 20 & Intraorbital & $\begin{array}{l}\mathrm{CT} \text {, enuclea- } \\
\text { tion left eye } \\
\text { and retroor- } \\
\text { bital mass, } \\
\text { Rt }\end{array}$ & $\begin{array}{l}\text { Mitosis } 3-4 / 10 \\
\text { HPF, pleo- } \\
\text { morphism }\end{array}$ & $\begin{array}{l}\text { Anorexia, } \\
\text { pneumonia, } \\
\text { comatose, } \\
\text { death due to } \\
\text { disease pro- } \\
\text { gression }\end{array}$ & 25 \\
\hline Figarella, 1992 & $45 / \mathrm{M}$ & TSS, DA, CT(2x) & Mitosis $1 / 2 \mathrm{HPF}$ & 8 & Vertebrae, lung & - & Not documented & Unknown & \\
\hline Kamphorst, 1992 & $45 / M$ & $\mathrm{CT}, \mathrm{Rt}$, chemo, Rt & Mitotic figures rare & 13 & $\begin{array}{l}\text { Pons, medulla } \\
\text { oblongata, } \\
\text { spinal cord }\end{array}$ & - & $\begin{array}{l}\text { Mitotic figures } \\
\text { rare }^{\mathrm{b}}\end{array}$ & $\begin{array}{l}\text { Death due to } \\
\text { disease pro- } \\
\text { gression }\end{array}$ & 13.02 \\
\hline Petterson, 1992 & $40 / \mathrm{M}$ & $\mathrm{CT}, \mathrm{Rt}, \mathrm{DA}$ & Mitotic figures & 5 & $\begin{array}{l}\text { Encasement caro- } \\
\text { tid bifurcation, } \\
\text { retro-orbital } \\
\text { space, cere- } \\
\text { brum, cerebello- } \\
\text { pontine angle, } \\
\text { nodule vertebral } \\
\text { artery }\end{array}$ & $\begin{array}{l}\text { CT, DA, CT, } \\
\text { chemo, CT, } \\
\text { chemo }\end{array}$ & $\begin{array}{l}\text { Pleomorphic, } \\
\text { invading over- } \\
\text { lying cerebral } \\
\text { tissue }\end{array}$ & $\begin{array}{l}\text { Death due to } \\
\text { disease pro- } \\
\text { gression }\end{array}$ & 8 \\
\hline Assies, 1993 & 63/M & CT, Rt, DA & Not documented & 7 & Cerebrum & $\begin{array}{c}\text { Surgery, DA, } \\
\text { Rt }\end{array}$ & Not documented & $\begin{array}{l}\text { Death due to } \\
\text { disease pro- } \\
\text { gression }\end{array}$ & 8 \\
\hline \multirow[t]{2}{*}{ Kasantikul, 1993} & $30 / F$ & DA & No mitotic figures ${ }^{b}$ & & $\begin{array}{l}\text { Pons, subarach- } \\
\text { noid space }\end{array}$ & - & No mitotic figures ${ }^{b}$ & $\begin{array}{l}\text { Pneumonia, } \\
\text { duodenal } \\
\text { ulcer, deep } \\
\text { vein throm- } \\
\text { bosis left leg }\end{array}$ & 0.17 \\
\hline & 22/M & $\mathrm{CT}, \mathrm{Rt}$ & Benign & 3 & Optic nerves & CT & $\begin{array}{l}\text { Mitotic figures in } \\
\text { large numbers }\end{array}$ & $\begin{array}{l}\text { Still alive at } \\
\text { publication }\end{array}$ & \\
\hline \multirow[t]{3}{*}{ Walker, 1993} & $32 / \mathrm{M}$ & $\begin{array}{l}\text { TSS, Rt, DA, } \\
\text { TSS(4x), CT }\end{array}$ & No mitotic figures & 5 & $\begin{array}{l}\text { Sphenoidal and } \\
\text { ethmoidal } \\
\text { sinuses, orbit, } \\
\text { liver, lungs, hilar } \\
\text { nods }\end{array}$ & $\begin{array}{l}\text { Chemo, TSS, } \\
125 \text { I implan- } \\
\text { tation, TSS, } \\
\text { DA, Octreo- } \\
\text { tide, TSS, Rt, } \\
\text { Chemp }\end{array}$ & No mitotic figures & Pneumonia & 9.5 \\
\hline & $48 / F$ & Rt, DA, TSS & No mitotic figures & 15 & $\begin{array}{l}\text { Vertebrae, sacro- } \\
\text { iliac joints, } \\
\text { femur }\end{array}$ & $\mathrm{DA}, \mathrm{Rt}$, chemo & No mitotic figures ${ }^{c}$ & Renal failure & 15.5 \\
\hline & 49/M & $\mathrm{CT}, \mathrm{Rt}$ & Benign & 2 & $\begin{array}{l}\text { Mediastinal lymph } \\
\text { node, lung }\end{array}$ & $\begin{array}{l}\text { DA, CT, Rt, } \\
\text { Octreotide }\end{array}$ & No mitotic figures & $\begin{array}{l}\text { Pulmonary } \\
\text { embolus } \\
\text { post-opera- } \\
\text { tively after } \\
\text { hip replace- } \\
\text { ment }\end{array}$ & 3.5 \\
\hline Long, 1994 & 70/M & TSS & No mitotic figures & 6 & Cerebrum & $\begin{array}{l}\text { CT, Rt, CT, Rt, } \\
\text { DA }\end{array}$ & No mitotic figures ${ }^{b}$ & $\begin{array}{l}\text { Still alive at } \\
\text { publication }\end{array}$ & \\
\hline
\end{tabular}




\begin{tabular}{|c|c|c|c|c|c|c|c|c|c|}
\hline $\begin{array}{l}\text { Author, year of } \\
\text { publication }\end{array}$ & $\begin{array}{l}\text { Age/ } \\
\text { sex }\end{array}$ & $\begin{array}{c}\text { Primary } \\
\text { treatment }^{\mathrm{a}}\end{array}$ & $\begin{array}{l}\text { PA primary } \\
\text { tumor }\end{array}$ & $\begin{array}{c}\text { Time interval } \\
\text { diagnosis - } \\
\text { metastases } \\
\text { (years) }\end{array}$ & Metastatic sites & $\begin{array}{l}\text { Treatment of } \\
\text { metastases }^{\mathrm{a}}\end{array}$ & PA metastases & $\begin{array}{c}\text { Cause of } \\
\text { death }\end{array}$ & $\begin{array}{c}\text { Time interval } \\
\text { diagnosis - } \\
\text { death (years) }\end{array}$ \\
\hline O’Brien, 1995 & $48 / \mathrm{M}$ & CT, Rt, DA & Benign & 5 & Cerebrum & CT & Mitoses frequent & $\begin{array}{l}\text { Still alive at } \\
\text { publication }\end{array}$ & \\
\hline Gollard, 1995 & $33 / F$ & $\begin{array}{c}\text { TSS, DA, TSS } \\
\text { biopsy, Rt }\end{array}$ & No mitotic figures & 12 & $\begin{array}{l}\text { Cheek pouch, } \\
\text { cerebrum, } \\
\text { pelvis, ovaries }\end{array}$ & $\begin{array}{l}\text { DA, surgery, } \\
\text { Rt, hyster- } \\
\text { ectomy, sal- } \\
\text { pingo- } \\
\text { oophorect- } \\
\text { omy, chemo }\end{array}$ & Mitosis $1-3 / \mathrm{HPF}$ & $\begin{array}{l}\text { Still alive at } \\
\text { publication }\end{array}$ & \\
\hline Saeger, 1995 & $59 / \mathrm{M}$ & $\mathrm{DA}, \mathrm{TSS}(2 \mathrm{x}), \mathrm{Rt}$ & Mitotic figures & 5 & Liver & Chemo, DA & Pleomorphic $^{c}$ & $\begin{array}{l}\text { Pulmonary } \\
\text { embolus }\end{array}$ & 6 \\
\hline Rockwell, 1996 & $50 / \mathrm{M}$ & TSS, CT, Rt, DA & Benign & 16 & Spinal intradural & $\begin{array}{l}\text { Gamma-knife } \\
\text { radiosurgery, } \\
\text { LT, Rt, DA }\end{array}$ & Mitotic figures & $\begin{array}{l}\text { Still alive at } \\
\text { publication }\end{array}$ & \\
\hline Bayindir, 1997 & $32 / F$ & DA, TSS & $\begin{array}{l}\text { Mitoses and } \\
\text { necroses, } \\
\text { pleomorphic }\end{array}$ & 0.08 & $\begin{array}{l}\text { Oculomotor nerve, } \\
\text { the optic fora- } \\
\text { men, encase- } \\
\text { ment carotid } \\
\text { artery, cere- } \\
\text { brum, spinal } \\
\text { cord }\end{array}$ & $\mathrm{CT}, \mathrm{LT}, \mathrm{CT}$ & $\begin{array}{c}\text { Mitoses and } \\
\text { necroses }\end{array}$ & $\begin{array}{l}\text { Death due to } \\
\text { disease pro- } \\
\text { gression }\end{array}$ & 0.25 \\
\hline Hurel, 1997 & $49 / F$ & TSS, Rt, DA & $\begin{array}{l}\text { Benign, p53 } \\
\text { positive }\end{array}$ & 5 & $\begin{array}{l}\text { Ethmoidal } \\
\text { sinuses, orbita, } \\
\text { temporal fossa, } \\
\text { pons, maxillary } \\
\text { antrum, sub- } \\
\text { mandibular } \\
\text { node }\end{array}$ & $\begin{array}{l}\text { CT, Rt, DA, } \\
\text { Octreotide, } \\
\text { chemo }\end{array}$ & $\begin{array}{l}\text { Pleomorphic, p53 } \\
\text { positive, Ki-67 } \\
\text { positive }\end{array}$ & $\begin{array}{l}\text { Tumor infarc- } \\
\text { tion or } \\
\text { hemorrhage, } \\
\text { coma }\end{array}$ & 7 \\
\hline \multirow[t]{6}{*}{ Pernicone, 1997} & $44 / F$ & TSS, Rt & Not documented & 3 & $\begin{array}{l}\text { Oral submucosa, } \\
\text { ovaries, myo- } \\
\text { metrium, pelvic } \\
\text { nodes }\end{array}$ & $\begin{array}{l}\text { Surgery, Rt, } \\
\text { chemo, DA }\end{array}$ & Not documented & $\begin{array}{l}\text { Still alive at } \\
\text { publication }\end{array}$ & \\
\hline & $34 / \mathrm{M}$ & DA, Rt, TSS & Not documented & 3 & Vertebrae, femur & Rt & Not documented & $\begin{array}{l}\text { Death due to } \\
\text { disease pro- } \\
\text { gression }\end{array}$ & 4 \\
\hline & $62 / M$ & $\mathrm{CT}, \mathrm{Rt}$ & Not documented & 3 & Cerebellum & $\begin{array}{l}\text { Rt, DA, } \\
\text { Surgery }\end{array}$ & Not documented & $\begin{array}{l}\text { Death due to } \\
\text { disease pro- } \\
\text { gression }\end{array}$ & 11 \\
\hline & $54 / F$ & TSS & Not documented & 1 & $\begin{array}{l}\text { Spinal subarach- } \\
\text { noid }\end{array}$ & Rt, DA, chemo & Not documented & $\begin{array}{l}\text { Death due to } \\
\text { disease pro- } \\
\text { gression }\end{array}$ & 3 \\
\hline & $37 / M$ & $\operatorname{TSS}(2 x)$ & Not documented & 6 & Lymph nodes & Rt & Not documented & $\begin{array}{l}\text { Death due to } \\
\text { disease pro- } \\
\text { gression }\end{array}$ & 7 \\
\hline & $64 / \mathrm{M}$ & TSS & Not documented & 6 & $\begin{array}{l}\text { Occipital lobe, } \\
\text { tentorium }\end{array}$ & Unknown & Not documented & $\begin{array}{l}\text { Still alive at } \\
\text { publication }\end{array}$ & \\
\hline
\end{tabular}




\begin{tabular}{|c|c|c|c|c|c|c|c|c|c|}
\hline $\begin{array}{l}\text { Author, year of } \\
\text { publication }\end{array}$ & $\begin{array}{l}\text { Age/ } \\
\text { sex }\end{array}$ & $\begin{array}{l}\text { Primary } \\
\text { treatment }^{\mathrm{a}}\end{array}$ & $\begin{array}{l}\text { PA primary } \\
\text { tumor }\end{array}$ & $\begin{array}{l}\text { Time interval } \\
\text { diagnosis - } \\
\text { metastases } \\
\text { (years) }\end{array}$ & Metastatic sites & $\begin{array}{l}\text { Treatment of } \\
\text { metastases }^{a}\end{array}$ & PA metastases & $\begin{array}{c}\text { Cause of } \\
\text { death }\end{array}$ & $\begin{array}{l}\text { Time interval } \\
\text { diagnosis - } \\
\text { death (years) }\end{array}$ \\
\hline Popadic, 1999 & $51 / \mathrm{M}$ & $\mathrm{CT}, \mathrm{DA}, \mathrm{Rt}$ & $\begin{array}{l}\text { Invasive prolacti- } \\
\text { noma, pleo- } \\
\text { morphism, no } \\
\text { mitotic figures }\end{array}$ & 4 & Spinal cord & $\begin{array}{l}\text { TSS, LT, Rt, } \\
\text { DA }\end{array}$ & $\begin{array}{l}\text { Pleomorphism } \\
\text { with rare mitotic } \\
\text { figures, tumor } \\
\text { cells in nervous } \\
\text { and fibrous tis- } \\
\text { sue }\end{array}$ & $\begin{array}{l}\text { Still alive at } \\
\text { publication }\end{array}$ & \\
\hline Arias, 2000 & $32 / \mathrm{M}$ & $\mathrm{DA}, \mathrm{CT}, \mathrm{Rt}$ & Mitosis & 1 & $\begin{array}{l}\text { Medulocerebral } \\
\text { angle, } \\
\text { vertebrae, } \\
\text { spinal epidural } \\
\text { space }\end{array}$ & $\mathrm{CT}$, Rt & $\begin{array}{l}\text { Mitosis; tumor } \\
\text { cells in subar- } \\
\text { achnoid space } \\
\text { and in venous } \\
\text { blood channels }\end{array}$ & Pneumonia & 3 \\
\hline Petrossians, 2000 & $43 / M$ & $C T, \operatorname{TSS}(2 \mathrm{x}), \mathrm{DA}$ & Not documented & 7 & $\begin{array}{l}\text { Spinal cord, rib, } \\
\text { mediastinum, } \\
\text { femur }\end{array}$ & $\begin{array}{l}\mathrm{CT}, \mathrm{Rt}, \mathrm{DA}, \\
\text { gamma-knife } \\
\text { radiosur- } \\
\text { gery }(4 \mathrm{x}), \\
\mathrm{CT}(2 \mathrm{x}), \mathrm{Rt}\end{array}$ & Not documented & $\begin{array}{l}\text { Death due to } \\
\text { disease pro- } \\
\text { gression }\end{array}$ & 15 \\
\hline Sironi, 2002 & $45 / M$ & $\begin{array}{l}\text { TSS, CT, TSS, Rt, } \\
\text { Sandostatin }\end{array}$ & Mitosis $1 / 20 \mathrm{HPF}$ & 9 & $\begin{array}{l}\text { Cerebrum, spinal } \\
\text { cord }\end{array}$ & $\mathrm{CT}(2 \mathrm{x}), \mathrm{Rt}$ & $\begin{array}{l}\text { Mitosis } 5-25 / 10 \\
\text { HPF, Ki-67 } \\
\text { positive, pleo- } \\
\text { morphic }\end{array}$ & $\begin{array}{l}\text { Pulmonary } \\
\text { embolism }\end{array}$ & 10.42 \\
\hline Vaquero, 2003 & $40 / M$ & $\mathrm{CT}, \mathrm{Rt}$ & Not documented & 14 & Cerebrum & Surgery & $\mathrm{Ki}-67<2 \%$ & $\begin{array}{l}\text { Still alive at } \\
\text { publication }\end{array}$ & \\
\hline Winkelmann, 2002 & $53 / \mathrm{M}$ & DA, TSS, CT, Rt & $\begin{array}{l}\text { Pleomorphism, } \\
\text { Ki-67 positive }\end{array}$ & 6 & $\begin{array}{l}\text { Orbita, foramen } \\
\text { magnum, } \\
\text { medulla oblon- } \\
\text { gata, spinal } \\
\text { cord, vertebrae }\end{array}$ & $\begin{array}{l}\text { DA, gamma- } \\
\text { knife radio- } \\
\text { surgery }(2 x)\end{array}$ & $\begin{array}{l}\text { Pleomorphic, Ki- } \\
67 \text { positive }^{\text {b }}\end{array}$ & $\begin{array}{l}\text { Renal failure, } \\
\text { lung edema, } \\
\text { death due to } \\
\text { disease pro- } \\
\text { gression }\end{array}$ & 7 \\
\hline Harinarayan, 2004 & $26 / M$ & CT, DA & Benign & 7 & Liver, gastric & DA, Octreo & Benign $^{c}$ & Unknown & \\
\hline Lamas, 2004 & $14 / \mathrm{M}$ & TSS, DA, Rt, CT & $\begin{array}{l}\text { Pleomorphism, } \\
\text { numerous mito- } \\
\text { tic figures }\end{array}$ & 6 & $\begin{array}{l}\text { Cerebrum, skull, } \\
\text { pulmonary } \\
\text { hilum, nodules } \\
\text { lungs, ribs, pel- } \\
\text { vis, spine }\end{array}$ & Chemo, DA & Mitotic figures ${ }^{c}$ & $\begin{array}{l}\text { Still alive at } \\
\text { publication }\end{array}$ & \\
\hline Noda, 2004 & $52 / F$ & TSS, CT, Rt, DA & Benign & 7 & $\begin{array}{l}\text { Cerebellum, } \\
\text { medulla oblon- } \\
\text { gata, spinal cord }\end{array}$ & $\begin{array}{l}\text { Gamma-knife } \\
\text { radiosurgery, } \\
\text { Rt, DA }\end{array}$ & $\begin{array}{l}\text { Pleomorphism, Ki- } \\
67 \text { positive }\end{array}$ & $\begin{array}{l}\text { Respiratory } \\
\text { arrest }\end{array}$ & 9 \\
\hline Uum Van, 2004 & $20 / F$ & $C T(2 x), D A$ & Not documented & 13 & Leptomeningeal & LT, DA & Low mitotic index & $\begin{array}{l}\text { Still alive at } \\
\text { publication }\end{array}$ & \\
\hline Vaishya, 2004 & $55 / F$ & $\mathrm{CT}, \mathrm{Rt}, \mathrm{DA}$ & Benign & 10 & $\begin{array}{l}\text { Encasement } \\
\text { internal carotis } \\
\text { artery, sphenoid } \\
\text { sinus }\end{array}$ & TSS, DA & $\begin{array}{l}\text { Mitosis } 2 / 10 \mathrm{HPF} \text {, } \\
\text { vascular inva- } \\
\text { sion, Ki-67 } \\
\text { positive }\end{array}$ & $\begin{array}{l}\text { Death due to } \\
\text { disease pro- } \\
\text { gression }\end{array}$ & 11 \\
\hline Crusius, 2005 & $47 / M$ & TSS, CT, Rt, DA & $\begin{array}{l}\mathrm{Ki}-672.80 \% \text { and } \\
4.40 \%\end{array}$ & 6 & Cerebrum & & $\mathrm{Ki}-674.45 \%^{\mathrm{c}}$ & Cardiac arrest & 6.02 \\
\hline
\end{tabular}




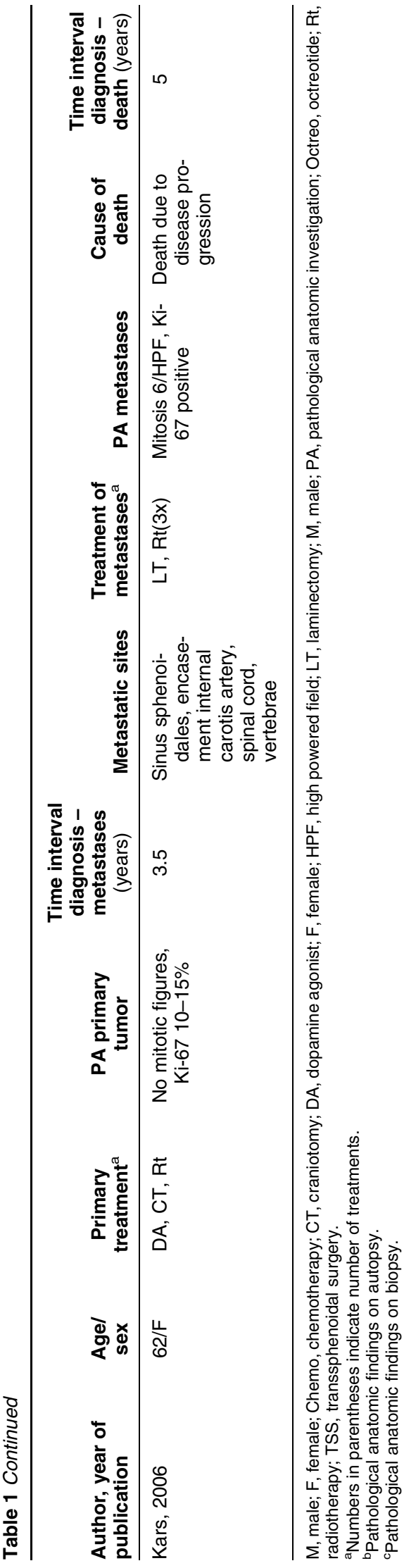

published cases was highly variable, with a median duration of 7 years, but ranging from 1 month to 20 years. Local recurrence after adenomectomy followed by repeated surgical interventions for local regrowth and extension of the pituitary tumor is frequently observed in malignant prolactinoma. Symptoms of prolactin hypersecretion rarely dominated the clinical picture of metastatic disease. However, symptoms of local compression at the metastatic sites were present in $73 \%$ of the cases. In some cases, metastases only manifested during autopsy $(14,18,20,21)$.

Intracranial metastases were reported in the frontal lobe $(7,9,12,18,19,22,23,27,33,34)$, parietaloccipital lobe $(6,22,29)$, temporal lobe $(10,18,24,28$, $33)$, cerebellum $(4,8,12,14,29,38)$, cerebellopontine angle $(5,18,31)$, brainstem $(17,20,28,38)$, and subarachnoid space $(9,14,20)$. Less commonly involved areas were the cranial nerves $(20,27)$ and the orbital space $(15,18,28,35)$. Extracranial metastases within the central nervous system involved the spinal cord $(8,11,13,14,17,26,27,29,30-33,35,38,39)$. Approximately $40 \%$ of the malignant prolactinomas were associated with systemic metastases in bone (10, $16,21,29,32,35,37)$, lymph nodes $(18,21,28,29$, $31,37)$, lung $(13,16,21,31,37)$, liver $(21,25,31$, $36)$, and, rarely, ovaries $(24,29)$.

Treatment of metastatic disease consisted of surgery in $69 \%$, radiotherapy in $54 \%$, and chemotherapy in $21 \%$ of cases. There is a case-to-case variability of the effect of chemotherapy on prognosis. At publication, three out of ten patients were still alive. Survival time of the remaining seven patients after being diagnosed with metastases was 2.1 years compared with 1.9 years for the whole cohort of patients. The mean time interval of diagnosis until death was 7.8 years compared with 8 years in all reported cases. Although, these data involve only a limited number of cases, we conclude that chemotherapy does not improve prognosis of malignant prolactinoma. In addition, $60 \%$ of the patients were treated with dopamine agonists. Only a minority of the patients was treated with octreotide $(8,21,28,36)$ or gamma-knife radiosurgery $(26,32,35,38)$. Survival in these patients was not different from the other patients.

Histological investigation of the metastatic lesions showed more often tissue with atypical parameters, compared with the results obtained from the primary tumor. Atypical features were present in $62 \%$ in the metastatic lesions versus $40 \%$ in the primary tumor.

The prognosis of malignant prolactinoma is poor. Survival after the onset of initial symptoms of prolactinoma is approximately 8 years, although there are patients who have survived for as long as 25 years. Only $60 \%$ of reported cases with a prolactin-secreting pituitary carcinoma survive more than 1 year after the development of metastases. It is presently difficult to estimate long-term survival in all patients since longterm follow-up has not been reported in most of these patients. 
Table 2 Summary of clinical features of malignant prolactinoma presented between 1981 and 2005 .

\begin{tabular}{ll}
\hline $\boldsymbol{n}=\mathbf{4 8}$ & \\
\hline Gender, male (\%) & 65 \\
Age, years & 43.6 (range 14-70 years) \\
Most prevailing symptomatology, number (\%) & Primary tumor \\
$\quad$ Hyperprolactinemia & $17(35)$ \\
Local compression & $34(71)$ \\
Metastatic sites (\%) & 75 \\
$\quad$ Intracranial & 33 \\
Extracranial & 40 \\
Extramedullary & 6.9 (range 1 month-20 years) \\
Mean time interval diagnosis - metastases (years) & 1.9 (range I week-8 years) \\
Mean time interval metastases - death (years) & 8.0 (range 2 months-25 years) \\
Mean time interval diagnosis - death (years) & 29 \\
Alive at publication (\%) & \\
\hline
\end{tabular}

Another feature indicative of a non-benign clinical course is the disappearance of prolactin-suppressive effects of treatment with DA. DA resistance, or an escape to the prolactin-suppressive effects, during treatment of prolactinoma is rare, but has been reported in the majority of patients with malignant prolactinomas (Table 3). Only six patients, however, including our case, were treated with cabergoline. When noncompliance is ruled out, this phenomenon is associated with dedifferentiation of the tumor and thus of malignant transformation. In our case, it is certainly remarkable because we found positive visualization of the pituitary tumor and the metastases by the epidepride scan (Fig. 5). Apparently, the tumor still expressed the D2 receptors because $\left[{ }^{123} \mathrm{I}\right]$ epidepride binds with high affinity to dopamine D2 receptors (46). Epidepride scintigraphy was only performed in our case and one previously reported case by Petrossians, et al. (32). Scintigraphy in the latter detected metastases in the spine, rib, mediastinum, and right femur.

Table 3 Histological, biochemical and therapeutic characteristics of the primary pituitary tumor and metastases.

\begin{tabular}{|c|c|c|c|}
\hline$n=48$, number $(\%)$ & $\begin{array}{l}\text { Primary } \\
\text { tumor }\end{array}$ & Metastases & Overall \\
\hline \multicolumn{4}{|c|}{ Histological classification } \\
\hline Typical & $18(37)$ & $8(17)$ & \\
\hline Atypical & $19(40)$ & $30(62)$ & \\
\hline Not documented & $11(23)$ & $10(21)$ & \\
\hline \multicolumn{4}{|l|}{ Response to DA } \\
\hline$D A$ resistance & & & $15(31)$ \\
\hline Cabergoline & & & $2(13)$ \\
\hline Others & & & $13(87)$ \\
\hline DA escape & & & $25(52)$ \\
\hline Cabergoline & & & $4(16)$ \\
\hline Others & & & $21(84)$ \\
\hline \multicolumn{4}{|l|}{ Therapy } \\
\hline Surgery & $46(96)$ & $33(69)$ & \\
\hline Radiotherapy & $38(79)$ & $26(54)$ & \\
\hline $\begin{array}{l}\text { Surgery and } \\
\text { radiotherapy }\end{array}$ & $38(79)$ & $20(42)$ & \\
\hline Chemotherapy & $1(2)$ & $10(21)$ & \\
\hline Dopamine agonist & $31(65)$ & $29(60)$ & \\
\hline
\end{tabular}

DA, dopamine agonists.
The metastases were treated with radiotherapy. In general, it is currently unclear how to translate these results in only two patients to the diagnostic value of this procedure in benign and malignant prolactinomas. The development of pituitary insufficiency within a time frame of several weeks is also consistent with tumor dedifferentiation and growth. The occurrence of pituitary insufficiency within such a short time interval is exceptional in pituitary adenoma.

In conclusion, malignant prolactinoma can present with unusual and atypical clinical manifestations. In the case of an invasive macroprolactinoma, these features, together with the development of resistance to dopamine agonists, should prompt the clinician to obtain histological information. In the presence of atypical indices, such as nuclear pleomorphism, numerous mitosis, and increased Ki-67 labeling index, the prolactinoma could be termed atypical to denote the potential of malignant transformation.

\section{References}

1 Kaltsas GA, Panagiotis N, Kontogeorgos G, Buchfelder M \& Grossman AB. Clinical review: diagnosis and management of pituitary carcinomas. Journal of Clinical Endocrinology and Metabolism 200590 3089-3099.

2 Ragel BT \& Couldwell WT. Pituitary carcinoma: a review of the literature. Neurosurgical Focus 200416 1-9.

3 Kaltsas GA \& Grossman AB. Malignant pituitary tumours. Pituitary 19981 69-81.

4 Martin NA, Hales M \& Wilson CB. Cerebellar metastasis from a prolactinoma during treatment with bromocriptine. Journal of Neurosurgery 198155 615-619.

5 Cohen DL, Diengdoh JV, Thomas DGT \& Himsworth RL. An intracranial metastasis from a PRL secreting pituitary tumour. Clinical Endocrinology 198318 259-264.

6 U SH \& Johnson C. Metastatic prolactin-secreting pituitary adenoma. Human Pathology 198415 94-96.

7 Gasser RW, Finkenstedt G, Skrabal F, Twerdy K, Grunert V, Mayr U, Frommhold H, Zur Nedden D, Feichtinger J \& Hofstaedter F. Multiple intracranial metastases from a prolactin secreting pituitary tumour. Clinical Endocrinology 198522 17-27.

8 Landgraf R, Rieder G, Schmiedek P, Clados D, Bise K \& Von Werder K. Hormone-active intradural spinal metastasis of a prolactinoma: a case report. Klinische Wochenschrift $198563 \quad 379-384$. 
9 Plangger CA, Twerdy K, Grunert V \& Weiser G. Subarachnoid metastases from a prolactinoma. Neurochirurgia $1985 \mathbf{2 8}$ 235-237.

10 Scheithauer BW, Randall RV, Laws ER, Kovacs KT, Horvath E \& Whitaker MD. Prolactin cell carcinoma of the pituitary. Clinicopathologic, immunohistochemical, and ultrastructural study of a case with cranial and extracranial metastases. Cancer 198555 598-604.

11 Von Werder K, Landgraf R, Oeckler R \& Rjosk HK. Treatment of prolactinoma. In Proceedings of the First Symposium of the European Neuroendocrine Association (ENEA), Dopamine and Neuroendocrine Active Substances, pp 73-82. Eds E Del Pozo \& E Fluckiger Basle: Academic Press, 1985.

12 Muhr C, Bergstrom M, Lundberg PO, Hartman M, Bergstrom K, Pellettieri L \& Langstrom B. Malignant prolactinoma with multiple intracranial metastases studied with positron emission tomography. Neurosurgery 198822 374-379.

13 Atienza DM, Vigersky RJ, Lack EE, Carriaga M, Rusnock EJ, Tsou E, Cerrone F, Kattah JG \& Sausville EA. Prolactin-producing pituitary carcinoma with pulmonary metastases. Cancer $1991 \mathbf{6 8}$ 1605-1610.

14 Popovic EA, Vattuone JR, Siu KH, Busmanis I, Pullar MJ \& Dowling J. Malignant prolactinomas. Neurosurgery 199129 127-130.

15 Berezin M, Gutman I, Tadmor R, Horowitz A \& Findler G. Malignant prolactinoma. Acta Endocrinologica $1992 \mathbf{1 2 7}$ 476-480.

16 Figarella-Branger D, Trouillas J, Morange I, Gambarelli D, Harle JR, Hassoun J, Grisoli F \& Pellissier JF. Prolactin pituitary carcinoma with multiple systemic metastases. Endocrine Pathology 19923 S11-S12.

17 Kamphorst W, Wolbers JG, Ponssen H \& Karim ABMF. Ectopic parasellar pituitary adenoma with subarachnoid seeding. Journal of Neurology, Neurosurgery and Psychiatry $1992 \mathbf{5 5}$ 73-74.

18 Petterson T, MacFarlane IA, MacKenzie JM \& Shaw MDM. Prolactin secreting pituitary carcinoma. Journal of Neurology, Neurosurgery and Psychiatry 199255 1205-1206.

19 Assies J, Verhoeff NPLG, Bosch DA \& Hofland LJ. Intracranial dissemination of a macroprolactinoma. Clinical Endocrinology 199338 539-546.

20 Kasantikul V, Boonjunwerwat D \& Suwangool P. Prolactin cell carcinoma of the pituitary. Journal of the Medical Association of Thailand 199376 230-237.

21 Walker JD, Grossman A, Anderson JV, Ur E, Trainer PJ, Benn J, Lowy C, Sonksen PH, Plowman PN, Lowe DG, Doniach I, Wass JAH \& Besser IM. Malignant prolactinoma with extracranial metastases: a report of three cases. Clinical Endocrinology 199338 411-419.

22 Long MA \& Colquhoun IR. Case report: multiple intra-cranial metastases from a prolactin-secreting pituitary tumour. Clinical Radiology 199449 356-358.

23 O'Brien DP, Phillips JP, Rawluk DR \& Farrell MA. Intracranial metastases from pituitary adenoma. British Journal of Neurosurgery $19959211-218$.

24 Gollard R, Kosty M, Cheney C, Copeland B \& Bordin G. Prolactinsecreting pituitary carcinoma with implants in the cheek pouch and metastases to the ovaries. Cancer 199576 1814-1820.

25 Saeger W, Bosse U, Pfingst E, Schierke G, Kulinna H, Atkins D \& Gullotta F. Prolaktinbildendes hypophysenkarzinom. Kasuistik eines ausserst seltenen metastasierenden tumors. Der Pathologe $199516354-358$.

26 Rockwell BH, Pica R, Raji MR, Dastur KJ, Altschuler EM \& Johnston JM. Intrathecal metastatic pituitary prolactinoma. American Journal of Roentgenology 1996167 1295-1296.

27 Bayindir C, Balak N \& Gazioglu N. Prolactin-secreting carcinoma of the pituitary: clinicopathological and immunohistochemical study of a case with intracranial and intraspinal dissemination. British Journal of Neurosurgery 199711 350-355.
28 Hurel SJ, Harris PE, McNicol AM, Foster S, Kelly WF \& Baylis PH. Metastatic prolactinoma: effect of octreotide, cabergoline, carboplatin and etoposide; immunocytochemical analysis of proto-oncogene expression. Journal of Clinical Endocrinology and Metabolism 199782 2962-2965.

29 Pernicone PJ, Scheithauer BW, Sebo TJ, Kovacs KT, Horvath E, Young WF, Lloyd RV, Davis DH, Guthrie BL \& Schoene WC. Pituitary carcinoma. A clinicopathologic study of 15 cases. Cancer $199779804-812$.

30 Popadic A, Witzmann A, Buchfelder M, Eiter H \& Komminoth P. Malignant prolactinoma. Case report and review of the literature. Surgical Neurology $1999 \mathbf{5 1}$ 47-55.

31 Arias M, Pereiro I, Requena I, Ventura M, Iglesias C \& Lema C. Malignant prolactinoma with intra- and extracranial metastasis: clinico-radiologic study. Anales de Medicina Interna $2000 \mathbf{1 7}$ 192-194.

32 Petrossians P, De Herder W, Kwekkeboom D, Lamberigts G, Stevenaert A \& Beckers A. Malignant prolactinoma discovered by D2 receptor imaging. Journal of Clinical Endocrinology and Metabolism $2000 \mathbf{8 5} 398-401$.

33 Sironi M, Cenacchi G, Cozzi L, Tonnarelli G, Lacobellis M, Trere D \& Assi A. Progression on metastatic neuroendocrine carcinoma from a recurrent prolactinoma. Journal of Clinical Pathology 2002 55 148-151.

34 Vaquero J, Herrero J \& Cincu R. Late development of frontal prolactinoma after resection of pituitary tumor. Journal of NeuroOncology $200364255-258$.

35 Winkelmann J, Pagotto U, Theodoropoulou M, Tatsch K, Saeger W, Muller A, Arzberger T, Schaaf L, Schumann EM, Trenkwalder C \& Stalla GK. Retention of dopamine 2 receptor mRNA and absence of the protein in craniospinal and extracranial metastasis of a malignant prolactinoma: a case report. European Journal of Endocrinology $200214681-88$.

36 Harinarayan CV \& Reddy K. Metastasising pituitary neuroendocrinal tumour with peptide secretion. Journal of the Association of Physicians of India 200452 74-75.

37 Lamas C, Nunez R, Garcia-Uria J, Salas C, Saucedo G, Estrada J, Parajon A \& Lucas T. Malignant prolactinoma with multiple bone and pulmonary metastases. Journal of Neurosurgery (Pediatrics) 2004101 (Suppl 1) 116-121.

38 Noda M, Suzuki R, Moriya M, Itokawa H, Asai J, Nagashima G \& Fujimoto T. A case of prolactin-secreting pituitary carcinoma and its histological findings. Brain Tumor Pathology 200421 149-154.

39 Van Uum SHM, Van Alfen N, Wesseling P, Lindert E, Pieters GFFM, Nooijen P \& Hermus ARMM. Massive reduction of tumour load and normalisation of hyperprolactinaemia after high dose cabergoline in metastasised prolactinoma causing thoracic syringomyelia. Journal of Neurology, Neurosurgery and Psychiatry 200475 1489-1491.

40 Vaishya A \& Tondon A. Malignant prolactinoma: is metastasis a must? Clinico-pathologic and immunohistochemical study of a case. Journal of Neurosurgical Sciences $2004 \mathbf{4 8} 37-41$.

41 Crusius PS, Forcelini CM, Mallmann AB, Silveira DA, Lersch E, Seibert CA, Crusius MU, Carazzo CA, Crusius CU \& Goellner E. Metastatic prolactinoma: case report with immunohistochemical assessment for p53 and Ki-67 antigens. Arquivos de Neuropsiquiatria $2005 \mathbf{6 3} 864-869$.

42 Isobe K, Ohta M, Yasuda S, Uno T, Hara R, Machida N, Saeki N, Yamaura A, Shigematsu N \& Ito H. Postoperative radiation therapy for pituitary adenoma. Journal of Neuro-oncology $2000 \mathbf{4 8}$ 135-140.

43 Vaudaux J, Portmann L, Maeder P \& Borruat FX. Orbital invasion by a pituitary macroadenoma without visual loss: case report and review of the literature. Eye 200317 1032-1034.

44 Thapar K, Kovacs K, Scheithauer BW, Stefaneanu L, Horvath E, Pernicone PJ, Murray D \& Laws ER. Proliferative activity and invasiveness among pituitary adonomas and carcinomas: an analysis using the MIB-1 antibody. Neurosurgery $1996 \mathbf{3 8}$ 99-106. 
45 Zornitzki T, Knobler H, Nass D, Hadani M \& Shimon I. Increased MIB-1/Ki-67 labeling index as a predictor of an aggressive course in a case of prolactinoma. Hormone Research 200461 111-116.

46 De Herder WW, Reijs AEM, Swart J, Kaandorp Y, Lamberts SWJ, Krenning EP \& Kwekkeboom DJ. Comparison of iodine-123 epidepride and iodine-123 IBZM for dopamine D2 receptor imaging in clinically non-functioning pituitary macroadenomas and macroprolactinomas. European Journal of Nuclear Medicine and Molecular Imaging 199926 45-50.
47 Hardy J \& Somma M. Acromegaly. Surgical treatment by transsphenoidal microsurgical removal of the pituitary adenoma. In Clinical Management of Pituitary Disorders, p 209. Eds WF Collins \& GT Tindall, New York: Raven Press, 1979.

Received 22 June 2006

Accepted 27 July 2006 